\title{
Mesures de vitesses radiales
}

\section{Accompagnement au sol du programme d'observation du satellite Hipparcos ${ }^{\star}$}

\section{Radial velocities. VIII. Ground based measurements for Hipparcos}

\author{
Ch. Fehrenbach ${ }^{1}$, M. Duflot ${ }^{2}$, C. Mannone ${ }^{2}$, R. Burnage ${ }^{1}$ et V. Genty ${ }^{2}$ \\ 1 Observatoire de Haute Provence, 04870 Saint Michel l'Observatoire, France \\ 2 Observatoire de Marseille, 2 Place Le Verrier, 13248 Marseille Cedex 04, France
}

Reçu le 8 octobre; accepté le 18 octobre 1996

\begin{abstract}
We publish 1879 radial velocities of stars distributed in 105 fields of $4^{\circ} \times 4^{\circ}$. We continue the PPO series (Fehrenbach et al. 1987; Duflot et al. 1990, 1992 and 1995), using the Fehrenbach objective prism method.
\end{abstract}

Key words: techniques: radial velocities - catalogues galaxy: kinematics and dynamics

\section{Introduction}

Nous continuons à publier les vitesses radiales $(V R)$ obtenues avec le petit prisme objectif (PPO) de Fehrenbach pour le programme d'accompagnement au sol du satellite Hipparcos. La technique des mesures est la même que celle utilisée précédemment (Burnage 1983 ; Fehrenbach et al. 1987). Rappelons brièvement que les mesures sont faites par corrélation entre des spectres de comparaison et les spectres à étudier, enregistrés au microphotomètre digitalisé (PDS) du Laboratoire d'Astronomie Spatiale de Marseille (LAS). Les étoiles de comparaison sont identiques à celles des publications précédentes (Duflot et al. 1992, 1995). Les clichés ont été obtenus pour la plus grande partie par G. Charvet et G. Traversa à l'Observatoire de Haute Provence. Pendant trois ans, G. Amieux a apporté une aide efficace à l'équipe des VR-PPO, en prenant des clichés en début et en fin de lunaison, avant l'arrivée et après le départ de G. Charvet

Send offprint requests to: M. Duflot

* Table 1 only available in electronic form at CDS via to cdsarc.u-strasbg.fr (130.79.128.5) or via http:// cdsweb.u-strasbg.fr/Abstract.html et G. Traversa et en préparant les mesures pour le microphotomètre du LAS (coordonnées des étoiles, spectres, etc...).

\section{Les résultats}

Nous publions les $V R$ de 1879 étoiles dans le Tableau 1 (disponible sour forme electronique seulement) ( $V R$ moyennes par champ) avec les mêmes conventions que dans les articles précédents (Duflot et al. 1990, 1992, 1995).

Les coordonnées $\alpha, \delta$ que nous publions sont celles calculées directement à partir des positions $x, y$ des spectres sur nos clichés, au moment de la mesure. Dans le cas éventuel d'une ambiguité sur l'identification, ce sont les données $\alpha, \delta$ qui sont donc sûres. Les types spectraux, les magnitudes $V$ et $B$ sont données à titre indicatif (sources diverses). En tête de chaque champ, un numéro de 4 chiffres correspond à l'heure en $\alpha$ (2 premiers chiffres) et aux degrés en $\delta$ ( 2 derniers chiffres) de l'étoile centrale. Deux de nos champs sont centrés sur des Selected Areas:

Champ $1945=$ SA 39

Champ $0600=$ SA 98.

Comme précédemment, nous avons vérifié que l'erreur standard $E, E=\sqrt{ } \frac{\sum \varepsilon^{2}}{n(n-1)}$ avec $\varepsilon=V R-\overline{V R} n=$ nombre de spectres mesurés, confirme l'erreur de $4 \mathrm{~km} \mathrm{~s}^{-1}$, pour quatre mesures (Fehrenbach et al. 1987).

Nos mesures au PPO étant relatives, nous avons recours pour étalonner nos champs, aux $V R$ de la littérature et éventuellement à des communications privées : à savoir, à la banque de données CORAVEL, par l'intermédiaire de M. Mayor, et aux résultats obtenus par S. Grenier, 
au spectrographe MARLY de l'Observatoire de Haute Provence.

En particulier, nous donnons ci-dessous, les $V R$ d'étoiles de nos champs, mesurées, à notre intention, par L. Prévot, avec le spectrophotomètre CORAVEL, à l'Observatoire de Haute Provence :

$\begin{array}{lrlll}\text { HD } & V R & \varepsilon & \mathrm{N} & \text { CHAMP } \\ & & & & \\ 53640 & 11,2 & 0,19 & 2 & 0718 \\ 54183 & 35,2 & 0,20 & 2 & 0718 \\ 54322 & -5,8 & & 1 & 0718 \\ 59014 & 187,4 & & 1 & 0735 \\ 59598 & -59,9 & & 1 & 0735 \\ 60986 & -36,8 & & 1 & 0735 \\ 64324 & 17,1 & & 1 & 0735 \text { Bis } \\ 64725 & 17,1 & & 1 & 0735 \text { Bis } \\ 64726 & 43,1 & & 1 & 0735 \text { Bis } \\ 65899 & -12,2 & & 1 & 0735 \text { Bis } \\ 74940 & -20,5 & & 1 & 0862 \\ 76362 & 4,3 & & 1 & 0862 \\ 80495 & 9,7 & & 1 & 0918 \\ 94426 & 5,4 & & 1 & 1032 \\ 100298 & -21,5 & & 1 & 1162 \\ 111132 & -4,0 & & 1 & 1210 \\ 129500 & -49,1 & & 1 & 1451 \\ 131194 & -47,0 & & 1 & 1451 \\ 178431 & -58,0 & & 1 & 1908 \\ 179870 & -17,2 & 0,33 & 6 & 1908 \\ 195608 & 10,7 & & 1 & 2049 \\ 197035 & -12,0 & & 1 & 2060 \\ 217478 & -18,3 & & 1 & 2222\end{array}$

La très grande vitesse de HD 59014, avait été détectée sur 2 clichés du champ 0735. Mais, le résultat de la mesure PPO n'est pas donné dans nos listes, car il n'est pas assez bien déterminé.

D'autre part, cinq étoiles se retrouvent dans deux champs différents de cette publication :

$\begin{array}{lcll} & \text { Champ 2040Bis } & \text { Champ 2040Ter } & \begin{array}{l}\text { Moyenne } \\ \text { HD 191636 }\end{array} \\ & -3 E=5.7(4) & -18 E=9.1(5) & \begin{array}{l}-11 E=5.9(9) \\ \text { Variable ? }\end{array} \\ & \text { Champ 2021 } & \text { Champ 2024 } & \\ \text { HD 192497 } & +6 E=3.0(4) & +6 E=9.1(3) & +6 E=3.8(7) \\ \text { HD 193348 } & -6 E=1.8(2) & -5 E=8.5(2) & -6 E=3.5(4) \\ \text { HD 193446 } & +6 E=1.8(4) & -7 E=6.5(2) & +1 E=3.4(6) \\ & & & \\ \text { HD 205947 } & \text { Champ 2161 } & \text { Champ 2164 } & \\ & 0 E=23.2(4) & -11 E=8.8(4) & -6 E=11.5(8) \\ & & & \text { Variable }\end{array}$

Les résultats de $V R$ sont disponibles au Centre de Données astronomiques de Strasbourg (CDS), ainsi que les valeurs individuelles obtenues par cliché.

Dans l'ensemble, les étoiles doubles visuelles connues que nous avons mesurées ont une trop forte différence de magnitude entre les composantes, ou un trop grand écartement pour que le spectre de la composante la plus brillante soit perturbé par la ou les autres composantes.

Font exception :

\begin{tabular}{|c|c|c|c|}
\hline HD & $\mathrm{mg}$ & $(\mathrm{CCDM})$ & $\rho$ \\
\hline 6886 & 7.3 & 7.5 & 0.7 \\
\hline 7102 & 7.9 & 8.9 & 1.1 \\
\hline 7854 & 8.0 & 9.0 & 0.3 \\
\hline 8187 & 7.4 & 7.9 & $0.4 \mathrm{ORB}$ \\
\hline 20682 & 8.4 & 8.5 & 7.0 \\
\hline 20698 & 8.5 & 8.9 & 1.1 \\
\hline 20947 & 7.6 & 8.3 & 1.0 \\
\hline 23406 & 9.5 & 9.7 & $0.3 \mathrm{ORB}$ \\
\hline 26882 & 8.0 & 8.4 & 0.4 \\
\hline 28544 & 8.5 & 9.2 & 1.3 \\
\hline 29695 & 8.6 & 8.6 & 1.2 \\
\hline 32652 & 8.7 & 8.7 & 0.9 \\
\hline 34948 & 8.6 & 8.6 & 0.2 \\
\hline 36263 & 7.8 & 8.3 & 1.2 \\
\hline 38068 & 8.5 & 8.7 & 0.5 \\
\hline 38179 & 6.7 & & \\
\hline 38411 & 9.1 & 9.1 & 0.1 \\
\hline 44696 & 9.2 & 9.7 & 0.9 \\
\hline 46048 & 7.2 & 8.2 & 4.7 \\
\hline 49294 & 7.4 & 7.9 & 0.4 \\
\hline 51473 & 9.1 & 10.0 & 0.3 \\
\hline 65738 & 9.1 & 9.1 & 0.5 \\
\hline 75680 & 9.0 & 9.0 & 0.2 \\
\hline 79096 & 6.4 & 7.2 & 0.2 \\
\hline 79127 & 8.1 & 8.6 & 1.8 \\
\hline 80082 & 8.2 & 8.3 & 0.2 \\
\hline 81899 & 8.2 & 9.1 & 0.5 \\
\hline 101150 & 6.8 & 7.8 & 2.1 \\
\hline 103659 & 6.8 & 7.4 & 0.4 \\
\hline 110280 & 7.6 & 8.1 & 1.4 \\
\hline 126126 & 9.2 & 9.2 & 0.7 \\
\hline 128941 & 7.4 & 7.7 & 0.6 \\
\hline 129600 & 8.0 & 8.0 & 2.0 \\
\hline 161016 & 7.6 & 7.9 & 0.7 \\
\hline 162405 & 8.2 & 8.7 & 0.3 \\
\hline 162670 & 8.4 & 8.8 & 0.3 \\
\hline 171728 & 8.1 & 8.2 & $0.3 \mathrm{ORB}$ \\
\hline 177904 & 7.2 & 7.9 & 8.0 \\
\hline 180160 & 7.8 & & \\
\hline 188871 & 8.6 & 8.6 & ORB \\
\hline 189451 & 8.6 & 9.0 & 0.2 \\
\hline 192659 & 7.1 & 7.3 & 0.8 \\
\hline 194558 & 7.6 & 7.6 & 0.1 \\
\hline 194882 & 7.2 & 7.4 & ORB \\
\hline 194907 & 8.3 & 9.3 & 0.7 \\
\hline 201672 & 8.1 & 8.5 & 2.5 \\
\hline 203991 & 7.5 & 8.4 & 0.5 \\
\hline 205599 & 8.6 & 8.8 & 0.5 \\
\hline 207990 & 7.5 & 8.5 & 4.3 \\
\hline 211405 & 8.2 & 8.2 & 0.7 \\
\hline 212153 & 8.5 & 9.2 & 0.3 \\
\hline $3973 / 4$ & 6.5 & 7.0 & $0.6 \mathrm{ORB}$ \\
\hline 214051 & 8.2 & 8.5 & 0.2 \\
\hline 216172 & 7.2 & 7.2 & 4.2 \\
\hline 216879 & 7.4 & 7.8 & 0.6 \\
\hline 223331 & 7.6 & 8.6 & 0.3 \\
\hline 223486 & 7.4 & 7.9 & $0.1 \mathrm{ORB}$ \\
\hline 224758 & 6.4 & & \\
\hline
\end{tabular}

Pour ces étoiles, la $V R$ publiée ici, peut correspondre à un mélange non décelé des deux spectres.

Remerciements. Nous remercions : le Directeur de l'Observatoire de Haute Provence, le Directeur et les Services du Laboratoire d'Astronomie Spatiale de Marseille où nous avons enregistré les clichés PPO, G. Amieux, G. Charvet, G. Traversa, J. Figuière qui assura efficacement le suivi du programme d'observations, S. Grenier, L. Prévot, M. Mayor, 
ainsi que les astronomes et techniciens qui ont fait les mesures Coravel, P. Figon et S. Martin du Service Informatique de l'Observatoire de Marseille, pour leur aide constante.

\section{Bibliographie}

Burnage R., 1983, Thèse, Université Aix-Marseille I Duflot M., Fehrenbach Ch., Mannone C., Genty V., 1990,
A\&AS 83, 251

Duflot M., Fehrenbach Ch., Mannone C., Burnage R., Genty V., 1992, A\&AS 94, 479

Duflot M., Fehrenbach Ch., Mannone C., Burnage R., Genty V., 1995, A\&AS 110, 177

Fehrenbach Ch., Duflot M., Burnage R., Mannone Cl., Peton A., Genty V., 1987, A\&AS 71, 275 